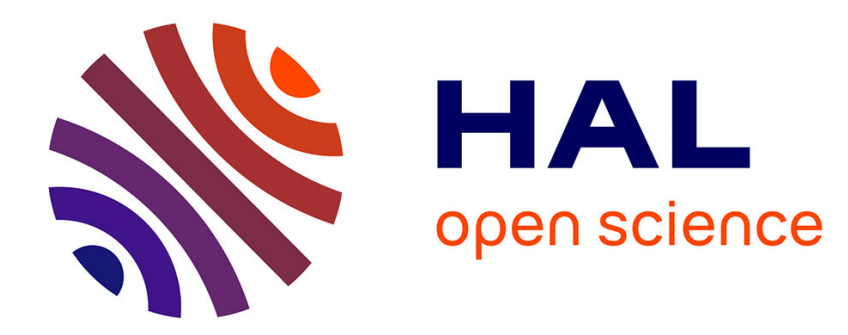

\title{
Le conseil souverain de Québec. Une institution de l'ancienne France pour le Nouveau Monde
}

\author{
Serge Dauchy
}

\section{To cite this version:}

Serge Dauchy. Le conseil souverain de Québec. Une institution de l'ancienne France pour le Nouveau Monde. Revue du Nord, 2015, 411 (97), pp.513-526. 10.3917/rdn.411.0513 . hal-02959164

HAL Id: hal-02959164

https://hal.univ-lille.fr/hal-02959164

Submitted on 6 Oct 2020

HAL is a multi-disciplinary open access archive for the deposit and dissemination of scientific research documents, whether they are published or not. The documents may come from teaching and research institutions in France or abroad, or from public or private research centers.
L'archive ouverte pluridisciplinaire HAL, est destinée au dépôt et à la diffusion de documents scientifiques de niveau recherche, publiés ou non, émanant des établissements d'enseignement et de recherche français ou étrangers, des laboratoires publics ou privés. 


\title{
Le conseil souverain de Québec
}

\section{Une institution de l'ancienne France pour le Nouveau Monde}

\author{
Serge DAUCHY
}

\section{Summary}

In April 1663, Louis XIVth decided to create a Sovereign Court of justice in New France, established in Québec. The royal edict of creation did not only organize the composition and functioning of the court, rationalizing and centralizing in particular the applied procedure and law. The edict also gives insight into the reasons and grounds of the creation of a high court of justice in the faraway and sparsely populated North American territories. The reasons were first political - assert the king's sovereignty over his overseas colonies - but appear also to be economical and commercial. The creation of a Sovereign Council in New France should therefor be considered primarily as the cornerstone of Colbert's mercantilist policy.

\section{Résumé}

Par un édit d'avril 1663, Louis XIV décida la création d'un conseil souverain pour la Nouvelle-France établi à Québec. L'édit royal de création n'organise par seulement la composition et le fonctionnement de la cour, en imposant en particulier une rationalisation et une centralisation de la procédure et du droit applicable. L'édit traduit également les raisons et motifs de la création d'une cour souveraine de justice dans les territoires nordaméricains éloignés et peu peuplés. Ces raisons sont d'abord politiques - affirmer la souveraineté du roi dans ses colonies d'Outre-mer - mais la création d'une cour souveraine est également motivée par des raisons économiques et commerciales. La justice apparaît en effet comme la pierre angulaire de la politique mercantiliste de Colbert. 
C'est par un édit donné à Paris au mois d'avril 1663 que Louis XIV fait connaître sa décision de créer un conseil souverain en Nouvelle-France ${ }^{1}$. L'établissement d'une cour souveraine à Québec intervient donc trois années après la création du conseil souverain de Roussillon et cinq années avant celui de Tournai. C'est surtout la première cour souveraine établie hors du Vieux Continent, la première expérience d'implantation d'une institution emblématique de l'ancienne France dans le Nouveau Monde. Toutefois, audelà d'une dénomination commune et d'une chronologie concomitante, l'établissement de conseils souverains au XVIIe siècle, en Métropole et en Outre-mer, s'inscrit dans des contextes différents et répond à des préoccupations et des logiques spécifiques dont les édits de création portent témoignage.

Malgré une structure et un plan comparables et en dépit de figures de style et de formulations lexicales inhérentes aux actes législatifs de l'époque, ces édits royaux ne sont pas coulés dans un moule préétabli. Ils sont, au contraire, rédigés en fonction des circonstances, tant geo-politiques et économiques qu'institutionnelles et juridiques. Tous les édits de création définissent la composition de la cour visée, les modalités de son fonctionnement et la délimitation de ses prérogatives, mais leur préambule précise également les raisons spécifiques qui ont motivé la décision royale, les conditions dans lesquelles intervient la création d'une nouvelle cour souveraine ainsi que les missions particulières qui lui sont éventuellement assignées. Ils participent ainsi à ce que l'on pourrait qualifier de politique communicationnelle de la monarchie. En effet, l'édit ne se résume pas à un commandement du souverain qui «de [sa] certaine science, pleine puissance et autorité royale» dispose et impose; c'est également un outil de communication au service des stratégies du producteur de la norme. Dans le cas présent, la monarchie cherche bien à nouer un lien - ce qui est le sens premier du terme «communiquer»- avec ses sujets, au premier rang desquels les membres du futur conseil, qui auront à enregistrer le texte et à mettre en œuvre la décision royale, et les différentes autorités concernées par l'établissement d'une cour d'appel souveraine exerçant par ailleurs d'importantes compétences réglementaires. Pour atteindre

\footnotetext{
${ }^{1}$ La Nouvelle-France désignait à l'origine l'ensemble des territoires de l'Amérique du Nord sous administration française. Da sa plus grande extension, avant le traité d'Utrecht de 1713, la Nouvelle-France comprenait cinq colonies possédant chacune une administration propre : le Canada, l'Acadie, la Baie d'Hudson, Terre-Neuve et la Louisiane.
} 
l'objectif fixé, le pouvoir royal ne peut se contenter du seul argument d'autorité. Il cherche dès lors à s'assurer, par l'explication et la motivation de sa décision, c'est-à-dire par une démarche pédagogique, une large adhésion des principaux destinataires du message royal, à savoir les justiciables du ressort de la juridiction nouvellement créée.

L'édit par lequel un conseil souverain est établi en la ville de Québec en $1663^{2}$ ne déroge pas à cette démarche programmatique, pas plus qu'il n'échappe à cette vocation communicationnelle. Le pouvoir royal a non seulement le souci de fixer l'organisation, la composition ainsi que les fondements du fonctionnement de la future cour, il prend également soin d'éclairer sa décision de transposer dans le nord du continent américain une juridiction souveraine.

1. Nous avons crû ne pouvoir prendre une meilleure résolution qu'en établissant une justice réglée et un conseil souverain dans le pays.

L'édit de création du Conseil souverain de la Nouvelle-France commence par rappeler l'histoire de la colonie nord-américaine depuis 1628. Cette date correspond à la reprise en main de la politique coloniale par Richelieu en sa qualité de grand maître, chef et surintendant de la navigation et commerce de France. Après l'échec de la politique consistant à nommer à la tête des territoires nord-américains des lieutenants-généraux ou vice-rois auxquels le roi cède le monopole de la traite des pelleteries, le cardinal organise en 1627 la Compagnie de la Nouvelle-France, plus connue sous le nom de Compagnie des Cent Associés. Cette nouvelle compagnie se voit concéder tout le Canada « en pleine propriété, fief et justice » ainsi que le monopole du commerce, en particulier celui des fourrures, à condition toutefois d'installer à ses frais 4.000 colons catholiques, de prendre en charge l'administration de la colonie et de soutenir les efforts de l'Eglise en vue de la conversion des amérindiens ${ }^{3}$. Bénéficiant d'un capital de départ consistant, la nouvelle compagnie entreprend parallèlement au développement de son activité commerciale - et cela pour la première fois depuis que Jacques Cartier a débarqué en 1534 sur les cotes de la Gaspésie et pris possession au nom du roi François Ier de toutes les terres présentes et circonvoisines - une véritable politique de peuplement. Elle

\footnotetext{
${ }^{2}$ Edits, ordonnances royaux, déclarations et arrêts du conseil d'Etat du roi concernant le Canada, vol. 1, Québec, 1854, p. 37-39.

${ }^{3}$ G.F. Stanley, «The policy of 'Francisation' as applied to the Indians during the Ancien Régime », dans Revue d'Histoire de l'Amérique française, III, 3 (1949), p. 333-348.
} 
organise l'installation d'un premier contingent de colons auxquels elle accorde « en fief et seigneurie » d'immenses étendues de terres le long du Saint-Laurent, à charge pour ces premiers colons de les concéder en censive aux futurs émigrants dont la Compagnie s'engage à prendre en charge la traversée. Administrée par un gouverneur choisi par la Compagnie et confirmé par le roi, la Nouvelle-France connaît à cette époque un réel essor qui se traduit par la fondation de Trois-Rivières, en 1635, et de Ville-Marie, à l'initiative de la Société Notre-Dame de Montréal pour la conversion des sauvages de la Nouvelle-France qui se fait concéder l'île de Montréal en 16404. Trois ans plus tard, toutefois, la Compagnie de la Nouvelle-France décide de céder son monopole sur le commerce des pelleteries à la Communauté des habitants de la colonie formée en société. Cette cession s'opère moyennement le paiement annuel d'un millier de peaux de castors et sous l'obligation de pourvoir aux charges financières de la Compagnie, cette dernière demeurant propriétaire du pays dont elle conserve également, formellement du moins, la direction. Car, en réalité, la monarchie intervient pour réorganiser les structures et l'administration de la colonie. Elle crée ainsi, en 1647, un conseil chargé de la régie de la traite des fourrures et de l'administration des finances de la NouvelleFrance. Composé à l'origine du gouverneur, du supérieur des Jésuites et du gouverneur particulier de Montréal, il est élargi en 1657 à quatre « conseillers », dont deux sont élus par les habitants de la ville de Québec et deux par ceux de Montréal et Trois-Rivières. Ce conseil dit «du gouverneur» a souvent été présenté comme l'ancêtre du Conseil souverain voire comme la première institution représentative du Canada, ce qui est probablement excessif puisque ses attributions concernaient essentiellement l'administration financière et la réglementation de la traite. Au cours des années qui suivent, les invasions iroquoises ruinent la jeune Communauté des habitants à tel point que l'existence-même de la colonie en est menacée. C'est, selon Gustave Lanctot ${ }^{5}$, ce qui aurait décidé la monarchie à intervenir. En 1663, la Compagnie de la Nouvelle-France est supprimée et le Canada, qui compte alors 2.500 colons, est réuni au domaine royal6.

\footnotetext{
${ }^{4}$ La concession de l'île de Montréal en faveur des « Messieurs du séminaire de Saint-Sulpice » du 17 décembre 1640 est ratifiée par le roi le 13 février 1644. Cf. Edits, ordonnances royaux, déclarations..., op. cit., vol. 1, , p. 24-26.

${ }^{5}$ G. Lanctot, L'administration de la Nouvelle-France, Montréal, 1971, p. 13.

${ }^{6}$ Edits, ordonnances royaux, déclarations..., op. cit., vol. 1, p. 31-32 : Abandon et démission de la Compagnie de la Nouvelle-France..
} 
Ce sont ces évènements que l'édit de création du Conseil souverain daté de cette même année rappelle en préambule7.

Les historiens, surtout canadiens, ne s'accordent pas sur les raisons qui ont poussé la monarchie à une reprise en main de la colonie nord-américaine ni sur celles qui l'auraient décidé à installer une cour souveraine dans ce territoire éloigné, difficilement accessible, peu peuplé et dont les pelleteries constituaient la seule ressource commerciale $^{8}$. Outre les difficultés financières d'une compagnie qui avait perdu une grande partie de ses actionnaires, certains ont prétendu que les excès de pouvoir des gouverneurs, et en particulier du baron d'Avaugour ${ }^{9}$, avaient été le facteur déterminant de l'intervention monarchique et ils avancent pour preuve que les premiers arrêts rendus par le Conseil souverain s'attachent à annuler les décisions prises par le dernier gouverneur sous le régime de la Compagnie de Nouvelle-France ${ }^{10}$. Il est suffisamment établi qu'il existait une forte animosité entre le gouverneur et François de Laval, premier évêque de Québec, débarqué au Canada en juin 1659 comme vicaire apostolique. Soucieux de faire valoir son autorité dans la colonie, tant vis-à-vis des Sulpiciens de Montréal que vis-à-vis des pouvoirs civils ${ }^{11}$, il s'était opposé d'emblée et publiquement aux orientations politiques et décisions du gouverneur. Ses rapports conflictuels avec les autorités coloniales s'expriment d'abord à travers des querelles de préséance. Il se cristallisent ensuite autour de la question de la vente d'eau-de-vie aux indigènes, un

\footnotetext{
${ }^{7}$ Edit de création du Conseil souverain de la Nouvelle-France, op. cit. : «La propriété du pays de la Nouvelle-France, qui appartenait à une compagnie de nos sujets, laquelle s'était formée pour y établir des colonies en vertu des concessions qui lui en auraient été accordées par le feu roi, notre très honoré seigneur et père de glorieuse mémoire, par le traité passé le 29 avril 1628, nous ayant été cédée par un contrat volontaire que les intéressés en la dite compagnie en ont fait à notre profit le 24e février dernier, ... ». On consultera sur l'histoire du Canada, en en particulier sur la période abordée ici, G. Vattier, Esquisse historique de la colonisation de la province de Québec (1608-1925), Paris, 1928 ; Cl. de Bonnault, Histoire du Canada français (1534-1763), Paris, 1950 ; L. A. de Grouly, Histoire du Canada français depuis la découverte, 4e éd., Montréal, 1962 ; M. Giraud, Histoire du Canada, 4e éd., Paris, 1966; M. Trudel, Initiation à la Nouvelle-France. Histoire et institutions, Montréal-Toronto, 1968 et Idem, Histoire de la Nouvelle-France, 4 t., Montréal, 1983-1997.

${ }^{8}$ Lédit de création évoque simplement « la distance des lieux trop grande pour pouvoir remédier d'ici à toutes choses »

${ }_{9}^{9}$ Pierre Dubois d'Avaugour est nommé gouverneur de la Nouvelle-France en 1661. Il est le dernier gouverneur à exercer sa charge sous l'autorité de la Compagnie des Cent-Associés : Dictionnaire biographique du Canada en ligne, http://www.biographi.ca/.

10 Voir E. Fabre-Surveyer, «La procédure sous le régime français», dans Rapport annuel de la Société d'histoire du Canada, 1932, p. 1-16.

${ }_{11}$ R. Dubois Cahall, The Sovereign Council of New France. A study in Canadian constitutional History, New York, 1915, p. 18sq.
} 
trafic que le gouverneur avait autorisé malgré l'opposition farouche du clergé qui y voyait la source des exactions commises par les populations autochtones et la cause principale des atteintes aux bonnes mœurs, à la religion et à l'ordre public. La position inflexible du gouverneur conduira le prélat à s'embarquer en 1662 pour la France afin de demander et d'obtenir finalement le rappel d'Avaugour. Il n'est pas exclu que la rencontre entre Mgr de Laval et Louis XIV fut également l'occasion d'évoquer l'avenir de la colonie nord-américaine, placée à présent sous l'autorité directe du roi, et d'aborder le projet de création d'un conseil souverain. C'est en effet au prélat, lorsque celui-ci retourna au Canada en 1663 accompagné du nouveau gouverneur de Mézy, que le roi confiera le soin d'apporter l'édit de création de la nouvelle juridiction souveraine. On a probablement trop peu insisté dans l'historiographie canadienne sur le rôle de Colbert, instigateur de la création du Conseil royal des finances en 1661. La reprise en main de la Nouvelle-France répondait autant à la volonté politique de Louis XIV d'affirmer la puissance du royaume sur la scène européenne - ce qui passait dorénavant par la maîtrise des mers et par le développement d'un empire colonial - qu'aux efforts déployés par Colbert pour mettre en place un mercantilisme à la française. Le premier empire colonial doit dès lors se comprendre, pour reprendre les propos d'Eric Wenzel, «à l'aune d'une volonté politique de fonder en Amérique des répliques de la sociétémère, avec des structures et institutions importées de la Métropole et principalement destinées à des populations expatriées » ${ }^{12}$. C'est dans ce sens qu'il convient d'interpréter le préambule de l'édit de création : « Nous avons estimé [...] que pour rendre le dit pays florissant et faire ressentir à ceux qui l'habitent le même repos et la même félicité dont nos autres sujets jouissent [...] il fallait pourvoir à l'établissement de la justice comme étant le principe, et en même temps un préalable absolument nécessaire pour bien administrer les affaires et assurer le gouvernement, dont la solidité dépend autant de la manutention des lois et de nos ordonnances que de la force de nos armes... ». Il n'est pas nécessaire d'insister sur l'importance qu'accordait le futur contrôleur général des finances (1665) et secrétaire d'Etat de la Marine (1669) à la centralisation de la justice et à l'uniformisation du droit. La prise en main de l'administration judiciaire constituait par ailleurs un mode habituel et directement compréhensible par les justiciables, de l'affirmation de la souveraineté du roi de France et elle demeurera encore aux XIX et

12 E. Wenzel, La justice criminelle en Nouvelle-France (1670-1760) : le grand arrangement, Dijon, 2012, p. 21. 
$\mathrm{XX}^{\mathrm{e}}$ siècles un puissant levier de la politique coloniale. Devant le morcellement des pouvoirs de justice et la multitude de juridictions, en France comme au Canada ${ }^{13}$, la volonté de plus en plus affichée de centralisation et d'unification de la justice passait dès lors par la création d'un parlement ou d'un conseil souverain, organisé sur le modèle des autres cours souveraines du royaume. L'affirmation de Louis Trénard selon laquelle « un pays était français quand ses habitants pouvaient porter une affaire devant le parlement », ou dans le cas présent devant un conseil souverain, prend ici, dans une contexte certes colonial, tout son sens ${ }^{14}$.

2. "... faisant garder autant qu'il se pourra la même forme de justice qui s'exerce dans notre Royaume, [Nous avons résolu] de composer le dit conseil souverain d'un nombre d'officiers convenables pour la rendre.

Introduit par la formule stéréotypée « Savoir faisons que Nous, pour ces causes et autres à ce nous mouvant... ", le dispositif de l'édit établit d'abord le siège du conseil souverain en la ville de Québec tout en se réservant la faculté de transférer, si nécessaire ou opportun, ce siège dans un autre lieu du pays, une précaution que d'autres édits, tel celui portant en 1668 établissement du conseil souverain de Tournai, prennent également. L'édit fixe ensuite la composition de la cour: "les sieurs de Mézy, gouverneur, représentant notre personne, de Laval, évêque de Pétrée, ou du premier ecclésiastique qui y sera, et de cinq conseillers [...] et d'un notre procureur [...]», un effectif restreint jugé suffisant pour répondre aux besoins d'un ressort certes étendu, mais guère peuplé de plus de 2.500 âmes. Les cinq conseillers seront choisis et nommés, conjointement et de concert par le gouverneur et l'évêque. Enfin l'édit charge également les deux principaux personnages de la colonie de désigner un greffier ou secrétaire chargé « de la conservation des minutes des arrêts, jugements et autres actes ou expéditions du conseil ». Tout juste débarqué en Nouvelle-France et ignorant des réalités locales, le gouverneur de Mézy laissa à l'évêque le soin de désigner les cinq conseillers et le

${ }^{13}$ En 1651, le gouverneur Jean de Lauzon dota la colonie d'une sénéchaussée. Un lieutenant général civil et criminel et un lieutenant particulier furent chargés de rendre la justice en première instance dans la ville de Québec et dans l'ensemble des territoires échappant à la compétence d'une juridiction seigneuriale établie. Des tribunaux seigneuriaux furent par ailleurs créés pour les territoires de Trois-Rivières et de Montréal. Le gouverneur connaissait en appel de toutes les décisions des justices seigneuriales. Voir P.-E. Audet, Les officiers de justice des origines de la colonie à nos jours, Montréal, 1986, p. 7-17.

${ }^{14}$ L. Trénard, Histoire des Pays-Bas français, Toulouse, 1972, p. 189. 
représentant du Ministère public lors de la première séance du Conseil, le 18 septembre 1663, séance au cours de laquelle l'édit de création fut solennellement enregistré. Son choix se porta sur cinq notables catholiques de la colonie : Louis Rouer de Villeray, Jean Juchereau de la Ferté, Denis-Joseph Ruette d'Auteuil, Charles Legardeur de Tilly et Mathieu Damours de Chauffours. Jean Bourdon fut choisi pour exercer la charge de procureur général et Jean-Baptiste Peuvret Demesnu fut nommé greffier et secrétaire.

Cette direction bicéphale est inédite et l'édit de création n'en fournit aucune explication. Il ne fait guère de doute que Mgr de Laval a usé de toute son influence pour obtenir du roi la coprésidence du conseil et le droit de nommer conjointement avec le gouverneur les membres de la cour. Mais on ne sait rien des arguments qui ont emporté l'arbitrage royal. S'agissait-il simplement de contrebalancer le pouvoir du gouverneur ou faut-il y voir un moyen de soutenir la conversion et la francisation des indigènes et peut-être aussi une volonté d'affirmer l'autorité de l'évêque face aux Sulpiciens et davantage encore face aux Jésuites ? Quoi qu'il est soit, la solution retenue présentait un risque en cas de désaccord entre les deux protagonistes. La rivalité ne tarda d'ailleurs pas à éclater au grand jour à propos de la reconduction des cinq conseillers. Conformément aux dispositions de l'édit de création, ceux-ci devaient en effet être « ... changés ou continués tous les ans selon qu'il sera estimé plus à propos et plus avantageux par les dits gouverneur, évêque ou premier ecclésiastique ». Un an à peine après l'installation du Conseil, le 24 septembre 1664, le gouverneur de Mésy prit l'initiative, sans en avoir référé à l'évêque, de ne pas reconduire Villeray et Tilly dans leurs fonctions et de nommer deux nouveaux conseillers. Ces graves dissensions, qui se creusèrent encore suite à la décision unilatérale du gouverneur de créer un office de substitut du procureur général et de nommer dans cette fonction Louis-Théandre Chartier de Lotbinière, devaient conduire le roi à intervenir une nouvelle fois. Le 23 mars 1665 il nomma Jean Talon intendant de justice, police et finances en Canada et deux mois plus tard celui-ci embarqua pour la Nouvelle-France en compagnie du nouveau gouverneur, Rémy de Courcelles. Talon était muni d'une commission qui lui transférait, conjointement avec le lieutenant général du roi de Tracy et le gouverneur le droit de nommer les conseillers du Conseil souverain. Quoi que l'édit de création ne mentionne pas l'intendant ${ }^{15}$, celui-ci s'imposa rapidement comme l'homme fort de la colonie et le véritable président du jamais la traversée. Cf. R. Roy, Les intendants de la Nouvelle-France, Ottawa, 1903. 
Conseil souverain. Malgré la cession du Canada à la Compagnie des Indes occidentales en mai $1664^{16}$, c'est bien depuis Versailles que la colonie est à présent dirigée et, comme l'atteste une correspondance soutenue ${ }^{17}$, c'est auprès de Colbert - nommé secrétaire d'Etat à la Marine en 1669 - que Jean Talon prend ses ordres.

3. Avons au dit conseil souverain donné et attribué [...] le pouvoir de connaître de toutes causes civiles et criminelles pour juger souverainement et en dernier ressort [...]

La principale mission assignée par le roi au nouveau Conseil souverain est celle « de connaître de toutes les causes civiles et criminelles pour juger souverainement et en dernier ressort». L'édit de création de 1663 précise à ce propos que le conseil devra «juger selon les loix et ordonnances de notre royaume et y procéder autant qu'il se pourra en la forme et manière qui se pratique et se garde dans le ressort de notre cour de parlement de Paris ». Pour D. Senécal, ce passage doit se comprendre dans le sens d'une « introduction formelle de la coutume de Paris en Canada » ${ }^{18}$. L'introduction de la coutume de Paris dans la colonie, à l'exclusion de toute autre, avait fait débat depuis l'établissement de la Compagnie de la Nouvelle-France en 1627. A plusieurs reprises, celle-ci avait affiché sa volonté d'imposer la primauté de la coutume de Paris dans l'ensemble des territoires qui lui avaient été concédés, mais sans jamais y parvenir. Le poids des colons normands installés en Nouvelle-France et surtout la compétence du parlement de Rouen, dont relevait la colonie nord-américaine jusqu'en 1663, constituaient autant de facteurs de résistance à une unification du droit et force est de constater que la grande majorité des contrats se conformaient plutôt, dans l'éventualité d'un litige, à la coutume du Vexin. Si donc l'intention du monarque avait été d'uniformiser et d'unifier le droit en imposant la coutume de Paris, la formulation de l'édit de création aurait sans nul doute été plus claire et plus explicite. C'est d'ailleurs ce

16 Edits, ordonnances royaux, déclarations..., op. cit., vol. 1, p. 40-48. L'édit par lequel est établie la Compagnie des Indes occidentales prévoit dans son article XXXI que «...où il sera besoin d'établir des conseils souverains, les officiers dont ils seront composés, nous seront nommés et présentés par les directeurs généraux de la dite compagnie, et sur les dites nominations les provisions seront expédiées ». Dans les faits, la Compagnie nomma son agent général au Canada comme conseiller supplémentaire, avec rang au-dessus du premier conseiller; les autres conseillers étant nommés, après le départ de Tracy en 1667, conjointement par l'intendant et le gouverneur, fonction exercée à partir de 1672 par le comte de Frontenac. En 1674, Louis XIV annula la charte de la Compagnie des Indes occidentales le pays fut définitivement intégré au domaine royal et administré par le secrétariat de la Marine.

${ }_{17}$ Centre des Archives d'Outre-mer d'Aix-en-Provence, correspondance ministérielle relative au Canada, série C11A.

18 D.H. Senécal, « Histoire de la coutume de Paris en Canada », dans Revue canadienne, 1864, p. 163-169 (p. 166). 
qui se passa un an plus tard. L'ordonnance de mai 1664 établissant la Compagnie des Indes occidentales est l'occasion d'imposer, officiellement et de manière très explicite cette fois, ce que Jacques Vanderlinden qualifie de «transplantation du droit français au Canada ${ }^{19}$. L'article XXXIII de l'édit dispose en effet: «Seront les juges établis en tous les dits lieux, tenus de juger suivant les loix et ordonnances du royaume, et les officiers de suivre et se conformer à la coutume de la prévôté et vicomté de Paris, suivant laquelle les habitans pourront contracter sans que l'on y puisse introduire aucune coutume pour éviter la diversité ».

C'est donc bien dans son sens littéral de règles «de procédure », qu'il convient de comprendre l'injonction faite par l'édit de création de respecter « la forme et manière qui se pratique dans le ressort de notre cour de parlement de Paris ». Cette formulation somme toute assez vague et la mention apportée par l'édit «selon les loix et ordonnances de notre royaume ", n'annoncent-elles pas l'œuvre codificatrice à venir ? Le projet de réformation de la justice, qui visait autant «la réduction en un seul corps d'ordonnances de tout ce qui est nécessaire pour établir une jurisprudence fixe» que «l'affirmation de la puissance législative qui réside en la personne seule du souverain » avait en effet été engagé par Colbert dès septembre 1661 comme l'atteste une lettre adressée à ce dernier par Pussort, le principal artisan de la future ordonnance civile ${ }^{20}$. Deux années plus tard, c'est-à-dire l'année de l'établissement d'un Conseil souverain en Nouvelle-France, le même Colbert donnait instruction aux commissaires départis dans les provinces d'enquêter sur les disfonctionnements de la justice, une véritable surveillance administrative des officiers de justice par les intendants étant alors ouvertement envisagée ${ }^{21}$.

19 J. Vanderlinden, «La réception des systèmes juridiques européens au Canada», dans Tijdschrift voor Rechtsgeschiedenis/Legal History Review, vol. LXIV (1996), p. 359-389. Voir également A. Gerin-Lajoie, "Introduction de la coutume de Paris au Canada ", dans Revue du barreau de la province de Québec, 1941, p. 61-65 et Y. Zoltvany, "Esquisse de la coutume de Paris », dans Revue d'Histoire de l'Amérique française, vol. 25, n 3, 1971, p. 365-384.

${ }^{20}$ P. Clément, Lettres, instructions et mémoires de Colbert, t. VI, Paris, 1869, p. 5, 6 et 368. Voir également Ph. Duval, La genèse du Code de procédure civile de 1806: à la frontière du droit et de la politique, thèse en Histoire du droit, Montpellier, 2007, p. 66-67.

${ }^{21}$ A. Astaing, « Le contrôle des juges par l'autorité administrative : l'exemple des justices royales de Bas-Languedoc au XVIIIe siècle », dans Critères du juste et contrôle du juge, Montpellier, 1996 (Recueil des mémoires et travaux publiés par la Société d'Histoire du droit et des institutions des anciens pays de droit écrit, fasc. XVII), p. 193-222. 
L'édit de création ayant rappelé, comme nous l'avons vu, la volonté royale de «[faire] garder autant qu'il se pourra la même forme de justice qui s'exerce dans notre Royaume », les grandes ordonnances louis-quatorzième ont tout naturellement trouvé leur application devant le conseil souverain de Nouvelle-France. En matière pénale, les travaux d'André Lachance ${ }^{22}$ et, plus récemment l'étude d'Eric Wenzel ${ }^{23}$, ont démontré que l'ordonnance criminelle de 1670 fut rapidement appliquée en Nouvelle-France, certes complétée par la jurisprudence du Conseil souverain et moyennant quelques adaptations que commandaient les conditions locales d'exercice. La mise en œuvre de l'ordonnance civile de 1667 suscita en revanche davantage de difficultés. Elle ne sera enregistrée par le Conseil souverain qu'en $1679^{24}$, après l'examen par le Conseil du roi d'une longue liste de quarante-sept remontrances formulées par la cour québécoise. La grande majorité de ces remontrances concerne les délais de procédure, nullement adaptés voire impossibles à adapter aux réalités canadiennes ${ }^{25}$. Outre « le grand éloignement de la colonie », le conseil souverain avait rappelé l'étendue des territoires nord-américains et les difficultés de déplacement auxquelles sont confrontés les gens de justice comme les justiciables. Les chemins praticables y sont quasiment inexistants, les habitations très éloignées les uns des autres et la seule voie de communication, le Saint-Laurent, qui relie Québec à Trois-Rivières et à Montréal - et qui est aussi la seule voie d'accès à la mer - est pris par les glaces une grande partie de l'année. C'est la raison pour laquelle la cour demanda et obtint que les délais, sans exception, « soient fixés par notre dit Conseil de Québec, ainsi qu'il le jugera raisonnable, selon la situation et la distance des lieux ». Toutefois, les adaptations consenties aux dispositions de l'ordonnance de 1667 ne sont pas uniquement imputables aux particularités géographiques et climatiques de la colonie nord-américaine. Elles doivent également pallier

\footnotetext{
${ }^{22}$ A. Lachance, La justice criminelle du roi au Canada au XVIIIe siècle, Québec, 1978 ; Idem, Crimes et criminels en Nouvelle-France, Montréal, 1984 et Idem, Délinquants, juges et Bourreaux en Nouvelle-France, Montréal, 2011.

${ }^{23} \mathrm{E}$. Wenzel, op. cit. A titre d'exemple, voir également S. Dauchy, « Trois procès à cadavre devant le Conseil souverain du Québec (1687-1708). Un exemple d'application de l'ordonnance de 1670 dans les colonies ", dans S. Dauchy et V. Demars-Sion (éd.), Juges et criminels. Études en hommage à R. Martinage, Lille, 2001, p. 37-49.

${ }^{24}$ Édits, ordonnances royaux, déclarations..., op. cit., p. 241 sq. : Édit du roi pour l'exécution de l'Ordonnance de 1667, juin 1679. Sur la procédure civile en Nouvelle-France, voir E. FabreSurveyer, op. cit. et J.A. Dickinson, Justice et justiciables. La procédure civile à la prévôté de Québec (1667-1759), Québec, 1982 (Les cahiers d'histoire de l'Université Laval, $\mathrm{n}^{\circ} 26$ ).

${ }^{25}$ S. Dauchy, «Nous voulons bannir les procès et la chicane qui sont la ruine des particulier et des Républiques. La réponse du Conseil souverain de Québec au problème des 'délais de procédure' (1663-1703), dans C.H. van Rhee (éd.), The Law's Delay. Essays on Undue Delay in Civil Litigation, Anvers-Oxford-New York, 2004, p. 83-91.
} 
un manque de juges expérimentés. Les premiers juges de la colonie, à tous les échelons d'une organisation judiciaire encore en construction, ont appris le métier sur le tas, souvent sans la moindre formation juridique. C'est également vrai pour les premiers magistrats du Conseil souverain. Villeray avait commencé sa carrière en 1654 comme secrétaire particulier du gouverneur Jean de Lauson avant de devenir lieutenant particulier de la sénéchaussée de Québec et commis du magasin de la Compagnie de la Nouvelle-France. Tilly, débarqué à Québec en 1636, s'était enrichi dans le commerce, obtenant en 1650 le monopole de la pêche à Tadoussac. Notable influent, il joua un rôle important dans l'organisation et l'administration de la Communauté des habitants avant de devenir conseiller. Chauffours, qui descendait d'une famille de parlementaires bretons, était arrivé au Canada en 1651 et s'était installé comme armateur. Il exerça durant plusieurs années la fonction de maire de la ville de Québec avant d'être nommé au Conseil souverain. La Ferté, embarqué pour le Nouveau Monde en 1634, s'était également enrichi dans le commerce avant de devenir conseiller. Quant au procureur général Jean Bourdon, il était arrivé dans la colonie en 1634 comme ingénieur-arpenteur et avait exercé ensuite différentes responsabilités telles que gouverneur intérimaire de TroisRivières, procureur-syndic de la ville de Québec et commis général de la Communauté d'habitants. Ces hommes possédaient une solide expérience de gestionnaire et d'administrateur, mais n'avaient été amenés à exercer la justice que dans le cadre ou en marge de leurs activités principales. A ses débuts, la juridiction souveraine s'apparente donc encore à ce que Bernard Durand qualifie de «justice de bâtiments de mer $»^{26}$. Ceci explique, entre autres, pourquoi la monarchie n'avait pas jugé à propos d'établir dans le pays des avocats et procureurs et elle entendait nullement ouvrir la boîte de Pandore. Le Conseil souverain luimême avait d'ailleurs estimé, comme on peut le lire dans le procès-verbal des modifications apportées à l'ordonnance de 1667, « qu'il est de l'avantage de la colonie de ne pas recevoir en ce païs avocats, procureurs ny praticiens » et parmi les raisons alléguées on retrouve, outre « la difficulté qu'il y a de faire des voyages et la pauvreté des habitans qui entreprennent à grands frais des procès sans y réfléchir », également « ...le peu d'expérience de la pluspart des juges $»^{27}$. Ce plaidoyer s'inscrivait largement dans les objectifs programmatiques définis par

${ }^{26}$ B. Durand, «Des justices de «bâtiments de mer », dans B. Durand et M. Badji (dir.), Le juge et l'Outre-mer, t. 5 : Justitia illiterata aequitate uti ? , Lille, 2010, p. 199-216.

${ }^{27}$ procès-verbal des modifications apportées par le Conseil du roi à l'ordonnance d'avril 1667, procès-verbal dans lequel sont reprises les motivations formulées par le Conseil souverain de Nouvelle-France : Édits, ordonnances royaux, déclarations..., op. cit., pp. 95 sq. Les seuls juristes (ou du moins personnes ayant une formation pratique du droit) présents en Nouvelle-France sont les notaires auxquels la Compagnie de la Nouvelle-France avait très tôt confié la rédaction de divers actes : contrats commerciaux, cession de monopoles, titres de propriétés et auxquels 
l'édit de création du Conseil souverain, à savoir « ... ôter autant qu'il se pourra toute chicane dans le pays de la Nouvelle-France afin que prompte et brève justice y soit rendue $»^{28}$. Et il ne s'agit pas là d'une simple figure de style. Consulté après la rétrocession par la Compagnie des Cent-Associés de la Nouvelle-France à la Couronne, le bureau des affaires coloniales avait fait rédiger un mémoire proposant divers moyens d'assurer la conservation et le développement du Canada. On s'y attarde longuement sur les questions de justice, préconisant autant qu'il se peut les modes extra-judiciaires de résolution des conflits et, si cela s'avère impossible, une justice où les formalités seraient réduites à leur plus simple expression. « Il faut », peut-on lire dans un mémoire de 1663, « prévenir les contestations et les estoufer dès leur naissance ; il faut entendre les parties par leur bouche sans admettre les artifices et les desguisemens ordinaires des procédures, les juger sur le champ et sans frais ; et s'il estoit absolument necessaire d'employer quelques instructions, se servir de celles qui ont esté ordonnéz pour les consulz qui sont les plus courtes et les moins à charge $»^{29}$. On ne pourrait être plus clair. Le pouvoir monarchique attend du Conseil souverain qu'il mette tout en œuvre «pour que les habitans puissent employer tout leur temps à la culture de la terre et à leur commerce » et, pour y parvenir, une mesure radicale s'impose : «bannir entièrement du pays les procès et la chicane qui sont la ruine des particuliers et des Républiques ». L'adaptation des règles de procédure, le mode de désignation des conseillers, l'absence d'auxiliaires de justice et l'unification du droit, toutes ces mesures - qui, pour reprendre les termes de l'édit de 1663, sont gage d'une « prompte et brève justice » - doivent concourir au double objectif assigné par Colbert à l'aventure coloniale : le renforcement de la place de la France sur l'échiquier politique international et le développement du commerce. Le roi le rappellera encore personnellement en 1680 à l'intendant Duchesnau: «les habitants de la

les habitants s'adressaient également pour établir des contrats de mariages et des testaments. Cf. J.-M. Augustin, « Les premiers contrats de mariage à Montréal de 1648 à 1664 et la coutume de Paris », dans La revue juridique Thémis, t. 30.1, 1996, p. 1-19. L'édit de 1663 confie d'ailleurs au Conseil souverain le soin de nommer les notaires et tabellions. A défaut d'avocats et de procureur, ces notaires se chargeront souvent de la rédaction des pièces de procédure indispensables et ils seront aussi consultés sur des questions de fond.

${ }^{28}$ Cette préoccupation est réitérée lorsque l'édit confie au Conseil souverain le soin d'appointer les personnes qui à Québec, Montréal et Trois-Rivières jugeront en première instance "sans chicane et longueur de procédure». G. Doutre, «La profession d'avocat et de notaire en Canada », dans Revue canadienne, 1873, p. 840-848 soutient au contraire l'idée selon laquelle Colbert aurait voulu bannir les avocats de la Nouvelle-France «parce qu'ils sont les principaux ennemis du système de coercition établi dans la colonie ».

${ }^{29} \mathrm{CAOM}, \mathrm{C}^{11 \mathrm{~A}} 2$, fo 41 . 
Nouvelle-France doivent être appliqués à la culture des terre, au commerce et manufacture et point divertis par les procédure en justice ${ }^{30}$.

Ces objectifs sont également rappelés par l'édit de création au moment d'aborder le pouvoir réglementaire reconnu au nouveau Conseil souverain. Outre une compétence en matière de police, publique et particulière, le conseil se voit en effet confier la mission « d'ordonner la dépense publique et de disposer de tout le trafic des pelleteries avec les sauvages, ensemble de tout le trafic que les habitants peuvent faire avec les marchands de ce Royaume ». Ce pouvoir règlementaire, que le Conseil souverain sera rapidement amené à partager avec l'intendant, tout comme les compétences judiciaires concourent à la réalisation des visées coloniales de la monarchie que sont le peuplement de la Nouvelle-France et son développement mercantile. Pierre angulaire du bon gouvernement, la justice contribue en effet, selon les termes de Colbert lui-même, «à peupler insensiblement la colonie [qui] pourra ainsi devenir considérable $\gg{ }^{31}$.

Conclusion

«C'est dans les colonies qu'on peut le mieux juger la physionomie du gouvernement de la métropole $»^{32}$. Cette affirmation d'Alexis de Tocqueville prend tout son sens au moment de la reprise en main de la Nouvelle-France par la monarchie en 1663, après une longue période de gestion privée finalement peu convaincante. Elle se vérifie en particulier à la lecture de l'édit de création d'un Conseil souverain à Québec - la première cour souveraine établie dans une colonie - qui apparaît comme véritable feuille de route de la politique coloniale louisquatorzième. Un examen rapide de l'édit de création du Conseil souverain de la NouvelleFrance peut en effet susciter l'impression d'un organisation judiciaire calquée sur le modèle métropolitain, d'un simple transposition des institutions et du droit de l'ancienne France dans le Nouveau Monde, certes adaptés au contexte coloniale et, en particulier, à son éloignement et aux difficultés de communication. Ces adaptations, comme l'écrit David Gilles, apparaissent alors « à visées pragmatiques » ou constituent « la poursuite d'intérêts divers »

\footnotetext{
${ }^{30} \mathrm{CAOM}, \mathrm{C}^{11 \mathrm{~A}} 5$, f० $209-217^{\mathrm{V}}, 2$ juin 1680.

${ }^{31}$ CAOM, C11A 2, fo $199,1664$.

${ }^{32}$ A. de Tocqueville, L'Ancien Régime et la Révolution, éd. J.-P. Mayer, Paris, 1967, p. 351.

${ }^{33}$ D. Gilles, «Les acteurs de la norme coloniale face au droit métropolitain : de l'adaptation à l'appropriation (Canada XVIIe - XVIIIe siècles) », dans Clio@Thémis. Revue électronique d'histoire du droit, $\mathrm{n}^{\circ} 4$ (2011).
} 
avec pour conséquence que «les acteurs [coloniaux] se trouvent dans une situation de soumission forte à la volonté normative métropolitaine ». L'introduction de l'ordonnance civile de 1667 et les adaptations consenties par le pouvoir central suite à des remontrances portant principalement sur les délais semblent en effet plaider dans ce sens. Toutefois, on ne peut réduire le mode particulier de désignation des conseillers - y compris l'absence de vénalité -, la volonté d'unification du droit - avec la coutume de Paris pour unique norme coutumière - ou encore l'interdiction sans cesse réitérée de permettre le recours à des avocats et des procureurs comme de simples «transplantations adaptées aux réalités locales ». La mainmise du gouvernement central ne fait aucun doute et, comme l'atteste la correspondance, c'est bien au secrétariat d'Etat de la Marine dominé par Colbert puis, à partir de 1715, au Conseil de la Marine que se prennent toutes les décisions importantes relatives aux colonies. Quant à l'intendant, homme de confiance du roi et de son ministre, il constitue à partir de l'arrivée de Jean Talon à Québec en 1665 le relais des décisions versaillaises et s'impose dès lors rapidement comme la véritable tête de l'administration canadienne et de sa justice.

Contrairement aux conseils souverains créés en Flandre, en Alsace et en Roussillon, la cour québécoise ne se présente pas comme un organe de conquête. Elle n'a pas pour objectif de briser les particularismes locaux ou de faire triompher les pratiques judiciaires françaises et le droit royal au détriment de normes et d'usages Pour autant, la politique monarchique ne se résume pas à une simple politique de transposition de règles et de cadres métropolitain, à charge des acteurs locaux - auxquels les outils juridiques et intellectuels semblent de surcroît faire défaut - de faire preuve de souplesse et d'inventivité à l'occasion de leur mise en pratique. Ne pourrait-on pas interpréter les dispositions de l'édit de création du Conseil souverain de la Nouvelle-France autrement qu'en termes de transposition, de pragmatisme voire d'acculturation? Ne serait-on pas confronté ici à l'expression d'une forme d'expérimentation de réformes ou de nouvelles structures et moyens juridiques qui, pour reprendre les termes de Bernard Durand, débouchent sur une « adaptation-expérimentation ${ }^{34}$ plus que sur une «imitation-adaptation »; une expérimentation judiciaire et administrative pensée d'ailleurs davantage en fonction des objectifs politiques et économiques assignés à

\footnotetext{
${ }^{34}$ A titre d'exemple, on citera l'idée évoquée dans la correspondance entre l'intendant Talon et le Ministre de la marine (1665) d'introduire au Canada des juridictions consulaires qui seraient chargées de trancher, de manière rapide et peu coûteuse, «tous les litiges civils ». Cf. S. Dauchy, «Stratégies coloniales et instruments judiciaires en Nouvelle-France (1663-1703), dans B. Durand et M. Fabre (dir.), Le juge et l'Outre-mer, t. 1 : Phinée le divin ou les leçons du passé , Lille, 2005, p. 207-225.
} 
cette première expérience coloniale que dans l'optique, plus hypothétique, d'une refonte du système métropolitain?

Serge Dauchy

Directeur de recherches au CNRS

Directeur du Centre d'Histoire Judiciaire (UMR 8025 CNRS - Lille 2)

1, Place Déliot 59000 Lille

tel. 03.20.90.74.43

serge.dauchy@univ-lille2.fr 